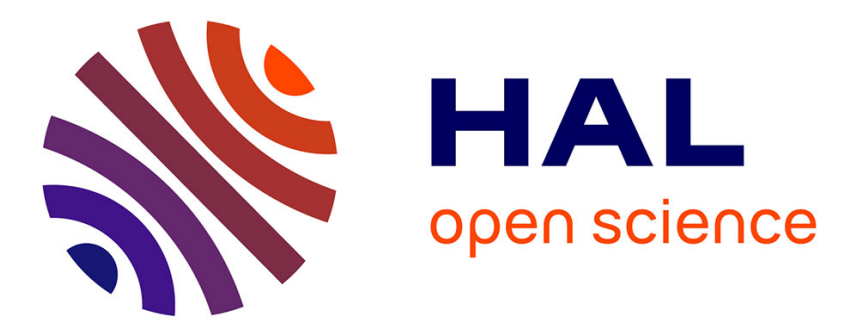

\title{
Compact and discriminative multi-object tracking with siamese CNNs
}

Claire Labit-Bonis, Jérôme Thomas, Frédéric Lerasle

\section{To cite this version:}

Claire Labit-Bonis, Jérôme Thomas, Frédéric Lerasle. Compact and discriminative multi-object tracking with siamese CNNs. IEEE International Conference on Pattern Recognition, Jan 2021, Milan (virtual), Italy. 10.1109/ICPR48806.2021.9412600 . hal-03125591

\section{HAL Id: hal-03125591 \\ https://hal.science/hal-03125591}

Submitted on 29 Jan 2021

HAL is a multi-disciplinary open access archive for the deposit and dissemination of scientific research documents, whether they are published or not. The documents may come from teaching and research institutions in France or abroad, or from public or private research centers.
L'archive ouverte pluridisciplinaire HAL, est destinée au dépôt et à la diffusion de documents scientifiques de niveau recherche, publiés ou non, émanant des établissements d'enseignement et de recherche français ou étrangers, des laboratoires publics ou privés. 


\section{Compact and discriminative multi-object tracking with siamese CNNs}

\author{
Claire Labit-Bonis \\ LAAS-CNRS \\ ACTIA Automotive \\ Toulouse, France \\ Email: clabitbo@laas.fr
}

\author{
Jérôme Thomas \\ ACTIA Automotive \\ Toulouse, France \\ Email: jerome.thomas@actia.fr
}

\author{
Frédéric Lerasle \\ LAAS-CNRS \\ Université UPS \\ Toulouse, France \\ Email: lerasle@laas.fr
}

\begin{abstract}
Following the tracking-by-detection paradigm, multiple object tracking deals with challenging scenarios, occlusions or even missing detections; the priority is often given to quality measures instead of speed, and a good trade-off between the two is hard to achieve. Based on recent work, we propose a fast, lightweight tracker able to predict targets position and reidentify them at once, when it is usually done with two sequential steps. To do so, we combine a bounding box regressor with a target-oriented appearance learner in a newly designed and unified architecture. This way, our tracker can infer the targets' image pose but also provide us with a confidence level about target identity.

Most of the time, it is also common to filter out the detector outputs with a preprocessing step, throwing away precious information about what has been seen in the image. We propose a tracks management strategy able to balance efficiently between detection and tracking outputs and their associated likelihoods.

Simply put, we spotlight a full siamese based single object tracker able to predict both position and appearance features at once with a light-weight and all-in-one architecture, within a balanced overall multi-target management strategy. We demonstrate the efficiency and speed of our system w.r.t the literature on the well-known MOT17 challenge benchmark, and bring to the fore qualitative evaluations as well as state-of-the-art quantitative results.
\end{abstract}

\section{INTRODUCTION}

Visual and online multiple object tracking (MOT) finds applications in fields such as videosurveillance, human-machine interaction and most recently self-driving cars. It consists in visually targetting and maintaining the identities of different objects in the video stream, as long as they remain in the frame. It belongs to a very active computer vision community and has historically been tackled through the tracking-bydetection paradigm. It implies to (i) detect objects on a frameby-frame basis and (ii) link these detections between frames to incrementally reconstruct targets' tracklets. The detector resets the tracker and prevents it from drifting, while the tracker compensates for detection artifacts. The MOT challenge [1] is a worldwide reference in the field. Based on the frameby-frame detections of three different and uneven off-theshelf detectors (DPM, Faster R-CNN and SDP), it consists in tracking multiple people in complex scenes e.g., static, moving, crowded, during nighttime, etc.

Siamese architectures are powerful similarity learners and can provide information about the resemblance between two inputs by feeding them to a twin-headed network sharing all or part of its weights between branches [2], [3]. Thanks to them, substantial progress has been made on the visual object tracking (VOT) task which consists in tracking a single object in a whole video, having as only input the bounding box coordinates of the object at time $t_{0}$. Siamese trackers take as inputs a patch, cropped around the object to track at time $t$, along with a larger search area at time $t+n$, and predict the object displacement within the search area. Such trackers like GOTURN [4], Siamese-RPN [5] and most recently SiamFC++ [6] took a leap forward both in terms of accuracy and speed on the VOT challenge [7]. While siamese structures are rarely extended to MOT for position prediction, they are commonly used to produce similarity scores between targets during the data association phase. However, very recent work using these techniques for position prediction showed state-of-the-art performance on the MOT challenge [8], [9].

Although very competitive, these techniques still remain slow due to their inner structure applying independant and non compact networks for position prediction and similarity learning. As our underlying ojectives relate to embedded applications, we place CPU consumption as a priority and propose to learn an all-in-one structure to perform tracking and reidentification at the same time, using a recent and fast EfficientNet [10] backbone network for feature extraction.

On another note, many recent approaches preprocess the supplied detections by re-injecting them in a detector trained on the MOT challenge dataset. Tracktor [11] uses a full Faster R-CNN architecture and artificially replaces the RPN outputs by the challenge detections before feeding them to the detector head. MOTDT [12] applies R-FCN on the entire image to refine each detection. This efficient strategy highlights that having a robust detector in the beginning is essential. However, it seems non intuitive within the challenge context to apply a second detector on top of the existing one. Neither does it provide for target-oriented features: it needs an extra network to extract reidentification characteristics, and ends up with a time consuming overall process. Given these insights, we rely on good prior inputs and focus our evaluations on the best detector of the benchmark to show state-of-the-art results without any preprocessing of the detections, while providing for both position and reidentification features in a compact siamese based architecture. 
In a nutshell, this paper aggregates the strengths of a fast siamese bounding box regressor for SOT coupled with a similarity head to perform one-shot identity propagation in a multi-object tracking framework. Frame by frame, our network takes image patches centered on tracklets as inputs, and produces coordinates regression along with reidentification score as outputs. In this way, our framework can infer the targets' image trajectories while handling their IDs frame by frame.

This paper contributions are four-fold:

- We present an unprecedented all-in-one compact siamese CNN architecture with a state-of-the-art and light-weight EfficientNet backbone structure to address multi-object tracking-by-detection by integrating both single object tracking and identity propagation at once, in an online MOT context.

- We propose a tracklet management strategy handling the current targets' tracklet pool and input detections with no need for additional preprocessing.

- We evaluate our framework on the well-known MOT17 challenge and exhibit state-of-the-art MOT performance while drastically reducing CPU cost.

\section{RELATED WORK}

Computer vision relies on an extremely active community and multi-object tracking specifically is a very competitive field. Based on frame-by-frame inferred detections, it consists in reconstructing the image trajectories of multiple targets moving in the scene. It deals with challenging situations e.g., occlusions, detection artifacts, targets look-alike or scene variability.

Tracklets construction can be performed online i.e., by sequentially processing information up to the current frame, or offline i.e., by dealing with both past and future information. Lots of nowadays applications tend to be mobile or automotive-oriented, they require fast and online processing: we place ourselves in this context and compare our work to the online submissions of the MOT17 challenge [1] (since its first 2015 version, this benchmark has become a reference in the field with $\sim 1000$ citations).

\section{A. Online MOT}

The generic online tracking-by-detection process can be summarized by Algorithm 1. While looping over all frames of a video sequence, it consists in (i) detecting targets in each frame i.e., their image coordinates $x, y, w, h$ (center, width and height), (ii) predicting the current position of already existing trajectories, and (iii) associating them with the aforementioned detections. New tracklets are created if all detections weren't assigned, and a tracklet state management strategy is usually applied afterwards to end spurious tracklets.

If detections are given as inputs to the challenge, approaches adopt different variants for position prediction, tracklet-detection association and tracklets state management.

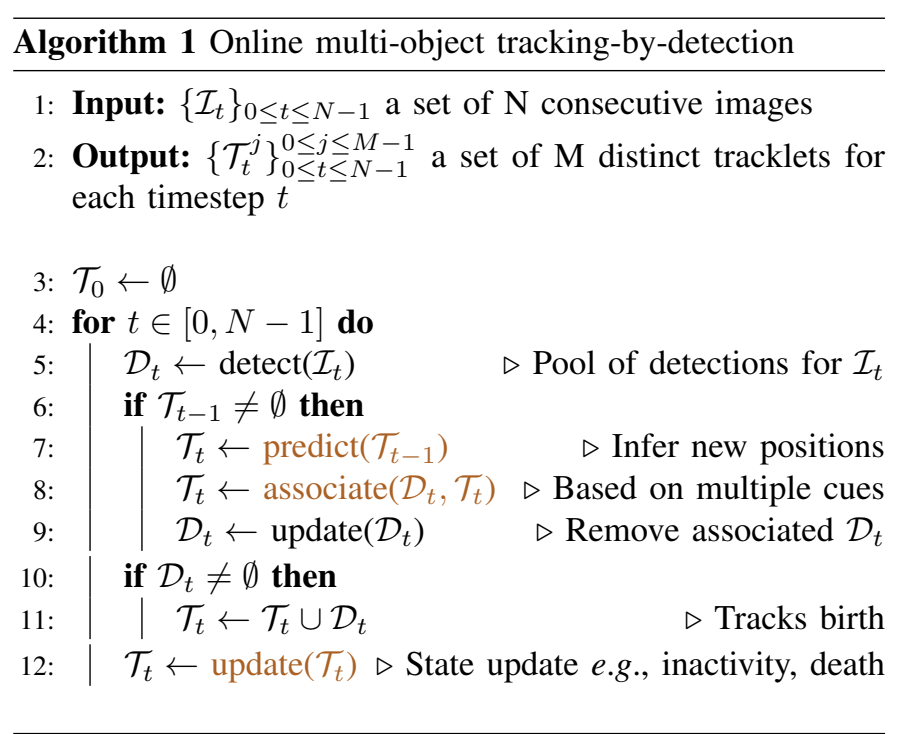

\section{B. Targets' position prediction}

From very simple approaches to more complex ones, a lot of methods have been proposed: SORT [13] applies a linear constant velocity model on each object to track, EAMTT [14] exploits a PHD Particle Filter, DeepSORT [15] and MOTDT [12] use a Kalman filter to estimate next tracklets states. Tracktor [11] uses the ROIPooling layer of a detector head to directly refine the bounding boxes. Most recently, FAMNet [8] and LSST [9] take advantage of siamese single object trackers to directly predict targets displacement based on their image crops. From a broader perspective, even if deep learning methods have become inescapable in computer vision applications, less than $20 \%$ of the online MOT17 submissions use such techniques for the position estimation part of the process. Yet, LSST [9] stands among the best on MOT17 and shows the interest of using visual CNN single object trackers in this multi-target context.

\section{Tracklet-detection association}

During the association phase between upcoming detections and existing tracklets, multiple cues can be considered to calculate a cost matrix for each potential match. The major one is related to the position of the objects to compare, but relying on appearance information helps at clarifying ambiguities between targets with close image positions. Whether it be through shallow descriptors [16] or deeply learned representations [12], [15], [17], most of the time it is done independently from the position prediction mechanism [9], [11], [12], [15], [18]. Amongst leading configurations, Tracktor [11] adds a heavy ResNet50 to extract target-specific reidentification features on top of its Faster R-CNN prediction structure, thus leading to a 1.5 FPS overall execution time; LSST [9] runs at 1.8 FPS by using an AlexNet-based structure for targets' position update, and a GoogLeNet Inception-v4 backbone for reidentification feature extraction. 


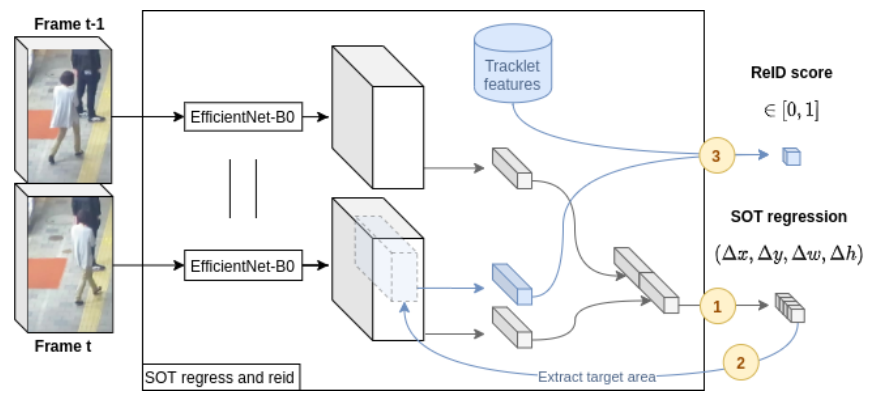

Fig. 1. For each current frame, all targets go through a SOT to (i) predict their displacement w.r.t their previous position, (ii) extract the target-specific embedding from the EfficientNet output feature map based on the predicted position offset, and (iii) compare it with the appearance embedding of the candidate tracklet and produce a reidentification score.

\section{Tracklets management strategy}

Updating the state of a tracklet i.e., its position, appearance, and managing its birth, death, inactivity is handled in various ways in the literature. Usually, position and appearance are replaced by the associated detections [12], [15], [19]. It is then common to consider a track active when it is assigned an incoming detection, and inactive when not associated in order to kill targets lost for too long [9], [11], [15]. By considering unfiltered detector inputs and setting up a balanced strategy, we propose to either rely on detector or tracker and keep targets active depending on the confidence of each element.

\section{Methodology}

Even if almost $60 \%$ of the MOT17 submissions use CNNs for reidentification, only a few take advantage of CNN-based techniques for predicting targets position. Above all, despite the performance shown by SOT within MOT [9], no previous work has been done to integrate a single light-weight CNN for both predicting targets position and producing reidentification features at once in this realtime multi-target context.

Furthermore, is it common to give full trust to the detector when it comes to updating tracklets status after the trackletdetection association phase; we put forward a balanced strategy allowing confident trajectories to keep living even when faced with spurious detections. We integrate multiple cues in our general framework, analyzed through an ablation study in section $\mathrm{V}$.

\section{A. Position prediction}

1) Single Object Track'n'Reid for MOT: We incorporate SOT-dedicated trackers in a multi-object framework by enriching them with identity awareness. Figure 1 describes how tracklets update their position and similarity scores at every timestep $t$, all-at-once through a single $\mathrm{CNN}$, before being faced with incoming detections. This tracklet prediction consists of three main parts:

- High-speed joint feature extraction. We take a patch from both previous and current frames, centered on the current target location at $t-1$. We call them target and search region patches. They are enlarged by a context factor $k$ as in [4], resized to [256 $\times 128]$ and fed to a siamese EfficientNet-B0 backbone for feature extraction, which shares its weights across both inputs.

- Motion prediction branch. On one side, we get two output volumes from EfficientNet corresponding to target and search patches inputs. We reduce the outcoming channel dimensions from 1280 to 128 , apply average pooling and concatenate outputs to finally send them to a 4-neuron $1 \times 1$ output layer predicting a $\Delta(x, y, w, h)$ shift vector, relatively to the search region width $w$ and height $h$. It is illustrated by the grey branch on figure 1 .

- Similarity branch. On the other side, we compute the cosine similarity between the current and predicted target appearance features. The appearance feature map is sliced from the feature extractor output volume of the search area, based on the predicted coordinate shift. It is then average pooled and transformed via $1 \times 1$ convolutions to finally calculate the cosine similarity with the target feature model. It is illustrated by the blue branch on figure 1.

2) Multi-task learning: In order to perform joint task learning, the network is trained with a combined regression/reid loss. As shown in figure 2, during training we feed the siamese network with SOT-dedicated inputs i.e., target and search patches at time $t$ and $t+1$, and batch multiple examples together. In parallel, we use them to construct positive and negative pairs later in the similarity branch. These pairs are sliced from the feature map centers at time $t$, average pooled, and used to calculate cosine similarity loss functions $\mathcal{L}_{\text {pos }}$ and $\mathcal{L}_{n e g}$ where positive (resp. negative) pairs scores must be close to 1 (resp. -1 ). The reidentification loss $\mathcal{L}_{\text {reid }}$ is the sum of cosine embedding losses for positive and negative pairs $\left(\mathcal{L}_{\text {pos }}+\mathcal{L}_{\text {neg }}\right)$

Regarding the position regression part of the network, both $t$ and $t+1$ feature extractor entire outputs are average pooled, concatenated and convolved to predict an $(x, y, w, h)$ offset which is compared to the groundtruth via L1 loss and stored in $\mathcal{L}_{\text {reg. }}$. In the end, the final loss can be written as in equation 1:

$$
\mathcal{L}=\mathcal{L}_{\text {reg }}+\frac{1}{2}\left(\mathcal{L}_{\text {pos }}+\mathcal{L}_{\text {neg }}\right)
$$

\section{B. Tracklet-detection association}

As mentioned in section II-C, at every online timestep each upcoming detection must either be associated to an existing trajectory, lead to a track birth or potentially be discarded. It is usually done by calculating an association cost between each possible detection-tracklet matching. The resulting cost matrix is then passed to a cost minimization solver, classically a Hungarian algorithm [20]. Different cues are usually considered to calculate the association cost, and the impact of each one is here explored with an ablation study in section V:

1) Intersection over Union: The overlap between bounding boxes $\mathrm{IoU}_{\mathcal{D}, \mathcal{T}}$ is a major discriminatory measure but it can lead to identity switches when two targets are close to each other. 

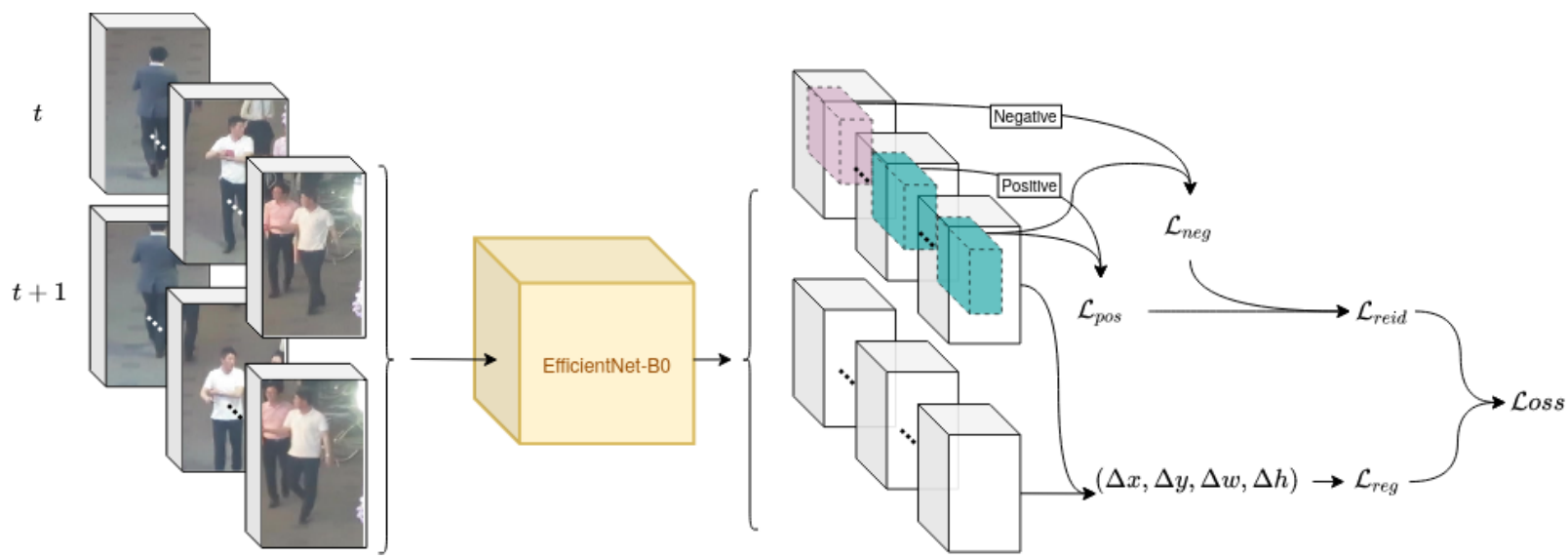

Fig. 2. Joint all-in-one training process of the full siamese SOT for regression and reidentification. For each target, two inputs at $t$ and $t+1$ are fed to an EfficienNet backbone for feature extraction. Multiple identities are batched together; we combine them to contruct positive and negative pairs in the batch. The feature extractor output volumes are used (i) in depth to extract sliced positive and negative pairs and calculate their cosine distance losses $\mathcal{L}_{\text {pos }}$ and $\mathcal{L}_{n e g}$, and (ii) between $t$ and $t+1$ to calculate the image coordinates displacement, thus the regression loss $\mathcal{L}_{\text {reg }}$. All losses are combined to produce a unique final loss.

2) ReID: To overcome this issue, we also apply the similarity branch of our network to the detections and compare their embedding with the targets ones to get the cosine similarity $\cos \mathcal{D}, \mathcal{T}$.

3) Detection score: We use the detector confidence scores $\mathcal{D}_{c f}$ lock, stock and barrel, arguing that based on good prior detections like SDP's, the use of raw inputs without filtering, altering or discarding information can be precious knowledge.

4) Target confidence: At every timestep, each tracklet updates an inner confidence score $\mathcal{T}_{c f}$ detailed in the next section and inspired by LSST [9].

We bring the $[-1,1]$ cosine similarity distance for reidentification back between 0 and 1 in order to finally multiply together $[0,1]$ range elements and get a normalized overall score. Combined together, we end up with a similarity score $\mathcal{S}_{\mathcal{D}, \mathcal{T}}$ between a detection $\mathcal{D}$ and a tracklet $\mathcal{T}$, thus an association cost $\mathcal{C}_{\mathcal{D}, \mathcal{T}}$ defined by equation 2 .

$$
\begin{aligned}
& \mathcal{S}_{\mathcal{D}, \mathcal{T}}=\mathcal{T}_{c f} \times \operatorname{IoU}_{\mathcal{D}, \mathcal{T}} \times \cos \mathcal{D}, \mathcal{T} \times \mathcal{D}_{c f} \\
& \mathcal{C}_{\mathcal{D}, \mathcal{T}}=1-\mathcal{S}_{\mathcal{D}, \mathcal{T}}
\end{aligned}
$$

\section{Tracklets management strategy}

At each frame, every tracklet updates its appearance embedding, confidence score and state depending on data association and prediction uncertainty. The integration of these elements in the overall multi-track algorithm is detailed at the end of the section.

1) Historical appearance embedding: As shown in figure 1, every tracklet compares the appearance sliced from the SOTpredicted position with its current appearance embedding. In order to avoid drifting, we only update these visual features when a match is found with a detection. This way, the tracklet reidentification confidence score states how much the prediction looks like the last associated detection.

Numerous approaches [9], [11], [15] use a list of the last matched appearance embeddings to overcome the issue of storing altered features $e . g$., if a target happens to be occluded or suddenly changes under varying lighting conditions, the appearance embedding is not crushed by the alteration and still has a stable representation in memory. The similarity score between a detection and a track embedding is therefore defined as a combination of the similarity scores among this list of stored features. Despite the aforementioned interest of this strategy, comparing tens of vectors in a combinatorial way e.g., in [11], [15], is time-consuming. As formulated in equation 3, we address this issue by storing only one appearance embedding $\mathcal{T}_{\text {feat }}$ for each track and updating it with the associated detection embedding $\mathcal{D}_{\text {feat }}$ weighted by a temporal memory factor $\tau_{m}$. This way, the comparison is fast and we still keep trace of appearance history.

$$
\mathcal{T}_{\text {feat }}=\left(1-\tau_{m}\right) \times \mathcal{T}_{\text {feat }}+\tau_{m} \times \mathcal{D}_{\text {feat }} \text { if } \operatorname{match}(\mathcal{D}, \mathcal{T})
$$

2) Tracklet confidence multi-score: In the vein of LSST [9], the tracklet confidence score formulated by equation 4 alternatively integrates information about track prediction or matched detection depending on the association phase outcome:

$$
\mathcal{T}_{c f_{t}}= \begin{cases}\frac{\left(\mathcal{T}_{c f_{t-1}}+\mathrm{IoU}_{\mathcal{D}, \mathcal{T}} \times \cos _{\mathcal{D}, \mathcal{T}} \times \mathcal{D}_{c f}\right)}{2} & \text { if } \operatorname{match}(\mathcal{D}, \mathcal{T}) \\ \mathcal{T}_{c f_{t-1}} \times \text { decay } \times \mathcal{T}_{\text {reid }}{ }^{k}, & \text { otherwise }\end{cases}
$$

- if a match is found between a detection $\mathcal{D}$ and a tracklet $\mathcal{T}$, the tracklet updates its previous confidence score $\mathcal{T}_{c f_{t-1}}$ with the product of the detection score, their overlap and their similarity score. Dividing the result by 2 as in equation 4 brings the score back to a $[0,1]$ range;

- if no match is found, we update the confidence score with a decay factor and the track reidentification score between prediction and current appearance features. As in [9], an exponent $k$ is applied to negatively impact uncertain tracks.

Where LSST only integrates IoU, we benefit from richer information like appearance similarity of match, prediction and detection confidence measure. 
3) Status update: The tracklet-detection association phase is not enough: it does not handle non-detections, and occlusions can still lead to inconsitency in tracklets. A mechanism is put in place to deactivate ambiguous trajectories and maintain alive non-associated tracks based on their SOT prediction confidence, thus finding the right balance between detection and tracking instead of only relying on the detector knowledge. The last step after prediction and data association then consists in updating the tracks as follows:

\section{a) Active/Inactive flag:}

- if a detection leads to track birth or is associated to an existing trajectory, this tracklet is set to active mode;

- if a track was active at time $t-1$ but is not assigned a detection at time $t$, it shifts to inactive mode only if the track confidence score $\mathcal{T}_{c f_{t}}$ drops below a certain threshold $\tau_{\text {active. }}$ This way, we also give trust to the tracker knowledge instead of only relying on the detector expertise as it is usually done;

- after data association, we perform Non-MaximumSuppression based on all targets confidence score with an IoU threshold $\tau_{n m s}$ to filter out occluded trajectories and transit them towards inactive mode.

A tracklet position is updated during prediction phase only if the track is active. This way, a drifting SOT will not stick to occluders or wander around when the target has exited the frame.

A two-step process is commonly applied during data association [11], [15] and consists in matching detections with active trajectories before looking at inactive ones. By using tracking confidence in the tracklet-detection assignment, inactive trajectories will implicitly lower the cost and be assigned after active ones. We thus apply a single-pass matching process to save processing time.

\section{b) Birth/Death management:}

- when a detection with a score $\mathcal{D}_{c f}$ is not assigned to any existing tracklet, it leads to the creation of a trajectory, only if $\mathcal{D}_{c f}$ is above a certain threshold $\tau_{\text {init }}$. Even if later in the association part we do consider every detection without any filtering, this threshold is important to avoid creating spurious false positive tracklets in the beginning and be confident about the appearance and position the SOT will have as a starting point;

- at birth, every tracklet is considered as a tentative. It is regarded as confirmed only after $n_{\text {init }}$ associations in a row. In this way, flickering detections e.g., two low confidence detections interleaved with a non-detection will not end up in the confirmation of a false track;

- if a track has not been associated to a detection $n_{\text {init }}$ consecutive times at birth, or if it has been inactive over than $n_{\text {wait }}$ successive frames, it gets killed. To this end, a counter $\mathcal{T}_{\text {counter }}$ is incremented as long as the tracklet remains inactive, and reset to 0 when a detection is matched. These two strategies are already used in the literature [15] and are efficient at reducing the number of false positives and identity switches.
All put together, the elements of prediction, data association and tracklets update can be summarized by Algorithm 2 .

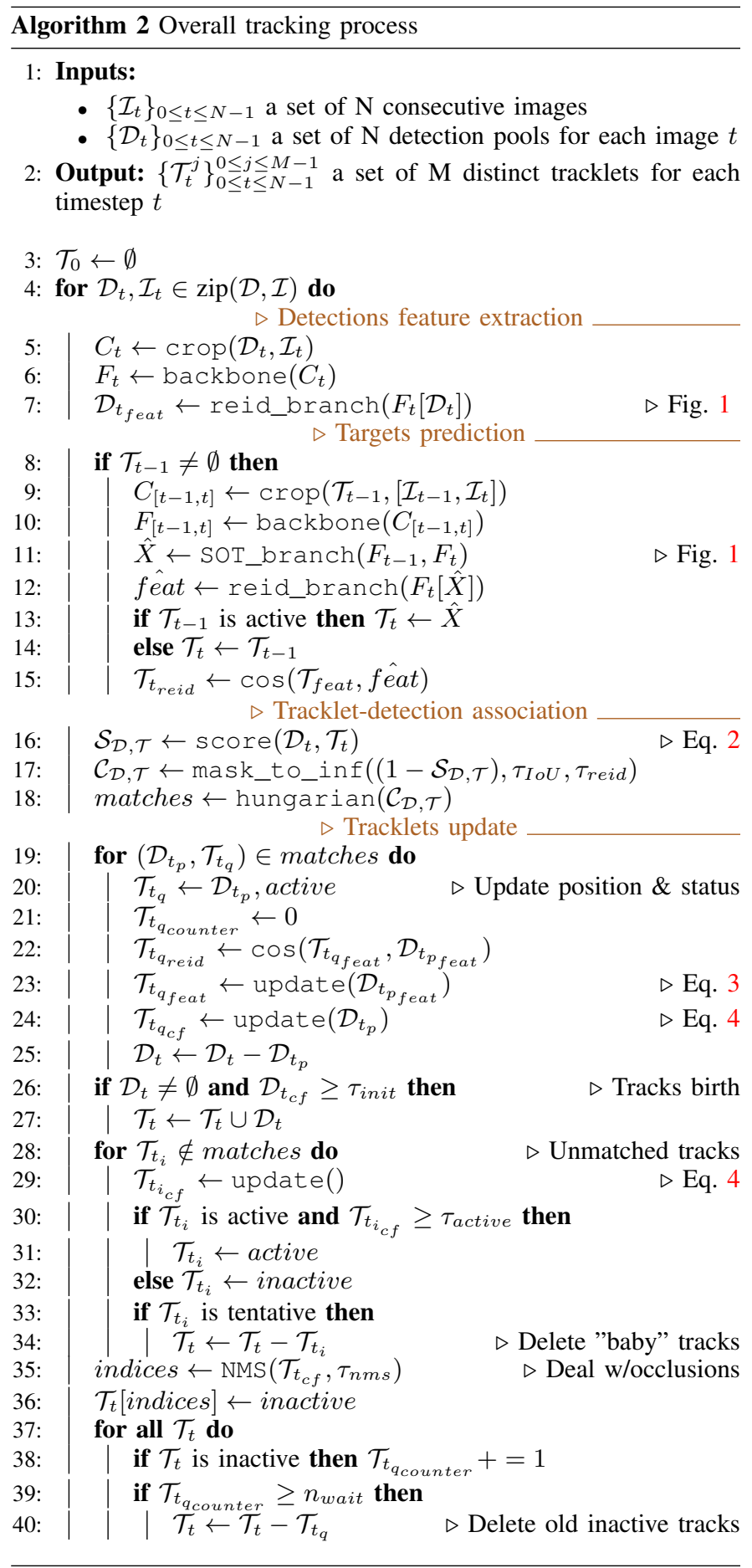

\section{IMPLEMENTATION AND LEARNING PHASE}

All evaluations are performed on an Intel Xeon E51620v4@3.50Ghz x 8 CPU with 16GB RAM and a NVIDIA Titan X (Pascal) GPU, with Python and Torch. 


\section{A. Dataset}

We evaluate ourselves on the MOT17 benchmark [1]. It consists of 14 pedestrian static and moving video sequences taken from different views, cut in half for training and testing. Among the whole dataset, 1280 distinct trajectories have been annotated following a strict protocol, and the output of three different frame-by-frame detectors are given as raw inputs to solve the multi-tracking task. As described in section I, we evaluate ourselves on the SDP detector and compare our results with the other MOT17 submissions for this detector.

\section{B. Training data generation}

1) SOT: As described in figure 2, we construct pairs of image crops centered on targets at time $t$ and cropped at the same location at time $t+1$. In order to get variability within the identities, we set to 3 the number of examples per track to generate i.e., for the same identified target, we take 3 different crops at different times and retrieve their corresponding $t+1$ crops. Within the same batch, we also retrieve 5 more identities with their 3 examples.

Because the displacement of a target between two successive frames is very small - thus close to zero, we also perform data augmentation by randomly shifting the crops taken from $t+1$ [4]. We end up with multiple [t, $t+1]$ pairs for each target, the first one being the real example, the others corresponding to the shifted augmented ones.

In the end, we have as input to the network a batch of 72 examples of $t$ and $t+1$ crops ( 6 identities, 3 examples per identity, 1 real $t+1$ and 3 augmented ones).

2) Similarity learning: Positive and negative pairs are built from the 72 crops at time $t$ available in a training batch. We construct 540 combinations of \{anchor, positive, negative\} triplets allowing us to calculate the positive cosine embedding loss for the \{anchor, positive $\}$ pairs and negative cosine embedding loss for the \{anchor, negative $\}$ pairs, the two categories being equally balanced.

\section{Hyperparameters}

All training parameters like context and scale factors or solver are the same as in [4]. The thresholds and tuned parameters of the overall process formulated in section III are optimized with SMAC [21] on training sequences and floored to the closest decimal value during inference. For clarity, we gather these hyperparameters, their values and description in Table I.

\section{EVALUATIONS AND ASSOCIATED ANALYSIS}

\section{A. Metrics}

As it is done in the benchmark, we evaluate our tracker with the CLEARMOT metrics [22]: false positives FP, false negatives FN, identity switches IDS and more importantly the multi object tracking accuracy MOTA which combines together these metrics and gives a relevant insight on the overall tracking performance. We also discuss time consumption performance i.e., the number of frames per second FPS.
TABLE I

HYPERPARAMETERS USED IN THE OVERALL MOT ALGORITHM.

\begin{tabular}{|rcl|}
\hline Param. & Value & Description \\
\hline \hline$\tau_{\text {init }}$ & 0.5 & Detection threshold for track birth \\
$\tau_{n m s}$ & 0.5 & IoU threshold for NMS \\
$\tau_{\text {IoU }}$ & 0.25 & IoU threshold for possible data association \\
$\tau_{\text {reid }}$ & 0.35 & Similarity threshold for possible data association \\
$\tau_{m}$ & 0.8 & Temporal memory weight factor for feature update \\
$\tau_{\text {active }}$ & 0.5 & Tracklet confidence threshold to keep active \\
decay & 0.98 & Tracklet confidence decay if not matched \\
$k$ & 2 & Confidence exponent used during update if not matched \\
$n_{\text {init }}$ & 3 & Time before a track gets confirmed \\
$n_{\text {wait }}$ & 20 & Inactive patience before killing a track \\
\hline
\end{tabular}

TABLE II

ABLATION STUDY SHOWING THE IMPACT OF THE TRACKLETS MANAGEMENT STRATEGY ELEMENTS FORMULATED IN SECTION III.

\begin{tabular}{|ccccc|ccc|c|}
$(\mathrm{A})$ & $(\mathrm{B})$ & $(\mathrm{C})$ & $(\mathrm{D})$ & $(\mathrm{E})$ & & & \\
\hline IoU & ReID & Classif. & Keep & Init & FP $\downarrow$ & FN $\downarrow$ & IDS $\downarrow$ & MOTA $\uparrow$ \\
\hline \hline$\checkmark$ & & & & & 4259 & 34314 & 1309 & 64.49 \\
$\checkmark$ & & & & $\checkmark$ & -1014 & +1004 & -267 & +0.24 \\
$\checkmark$ & & & $\checkmark$ & $\checkmark$ & +1693 & -1187 & -220 & -0.25 \\
$\checkmark$ & $\checkmark$ & & $\checkmark$ & $\checkmark$ & -1152 & +994 & -307 & +0.41 \\
$\checkmark$ & $\checkmark$ & & $\checkmark$ & $\checkmark$ & +191 & -802 & -263 & +0.78 \\
\hline \hline$\checkmark$ & $\checkmark$ & $\checkmark$ & $\checkmark$ & $\checkmark$ & -462 & -325 & -297 & +0.96 \\
\hline
\end{tabular}

\section{B. Ablation study}

The following ablative analysis has two important purposes:

- confirm the added value of each single component described in section III during tracklet-detection association (Eq. 2) and tracklet confidence score update (Eq. 4);

- find the optimal tracking performance configuration.

As described in section III, we use different information for calculating both tracklet-detection association costs and tracklet confidence scores: intersection over union (A) IoU, reidentification measure of the prediction/association (B) ReID, detection confidence score (C) Classif. When we say that one of them is not used, we mean both in the association cost and tracklet confidence score. We also analyze the impact of two tracklets management strategies: keeping a confident track active even if it is not associated to any detection ((D) Keep) and confirm a track only if associated to a detection $n_{\text {init }}$ times in a row after its creation ((E) Init).

In table II, IoU is used as a baseline to calculate the gain of each element.

1) Wait at init: $(\mathrm{A}+\mathrm{E})$ Waiting for multiple associations before confirming a tracklet shows a gain to the baseline of +0.24 on the MOTA. Looking at table II, we note that it is mainly due to the gain over identity switches which can be explained by the false positives drop down leading to less ambiguous situations.

2) Complementary ReID and keep active: An interesting outcome lies in the use of reidentification and keep active strategy separately and then in a complementary way.

$(A+B+E)$ On the one hand, we note that reidentification alone with IoU and initial patience simultaneously improves false positives and identity switches, thus following the expected behavior when adding appearance information as extra 
TABLE III

COMPARISON OF ONLINE CNN-BASED MOT17 SUBMISSIONS ON THE TEST SET FOR THE SDP DETECTOR.

\begin{tabular}{|c|c|r|r|r|r|c|c|c|c|c|c|}
\hline Rank & Year & Conf. & Method & MOTA $\uparrow$ & MT $\uparrow$ & ML $\downarrow$ & FP $\downarrow$ & FN $\downarrow$ & IDS $\downarrow$ & FPS $\uparrow$ & Hardware \\
\hline \hline 1 & 2020 & arXiv & UnsupTrack & $61.99 \%$ & 217 & 248 & 5986 & 64860 & 651 & 2 & GTX 1080Ti \\
2 & 2019 & arXiv & LSSTO [9] & $61.63 \%$ & 194 & 241 & 7285 & 64091 & 792 & 1.8 & GTX 1060 \\
3 & 2020 & IEEEAccess & YOONKJ & $61.11 \%$ & 225 & 248 & 9468 & 62895 & 788 & 3.4 & GTX 1080Ti \\
5 & 2018 & AVSS & HAM_SADF & $60.30 \%$ & 212 & 251 & 7177 & 66729 & 759 & 5 & Titan X \\
6 & 2019 & arXiv & DEEP_TAMA & $59.95 \%$ & 215 & 246 & 8445 & 66103 & 779 & 1.5 & Titan X \\
7 & 2019 & ICCV & FAMNet [8] & $59.16 \%$ & 198 & 233 & 4822 & 70900 & 1097 & 0.6 & Titan X \\
\hline \hline $\mathbf{9}$ & $\mathbf{2 0 2 0}$ & & Ours & $\mathbf{5 8 . 4 8 \%}$ & $\mathbf{1 9 8}$ & $\mathbf{2 3 4}$ & $\mathbf{8 5 8 5}$ & $\mathbf{6 8 1 6 8}$ & $\mathbf{1 3 2 8}$ & $\mathbf{1 0 . 4}$ & Titan X \\
\hline \hline 10 & 2018 & ICME & MOTDT [12] & $58.39 \%$ & 177 & 241 & 6317 & 71005 & 941 & 18.3 & GTX 1060 \\
11 & 2020 & IJCAI & GSM_Tracktor & $58.34 \%$ & 192 & 256 & 5772 & 72050 & 537 & 8.7 & Titan X \\
15 & 2019 & arXiv & GMPHD_Rd & $57.15 \%$ & 230 & 200 & 15661 & 63225 & 1686 & 20.4 & GTX 1050 \\
16 & 2020 & AAAI & DASOT [23] & $57.13 \%$ & 217 & 213 & 13205 & 65320 & 2102 & 9.1 & Titan X \\
17 & 2019 & TM & MTDF & $57.01 \%$ & 190 & 223 & 10183 & 68898 & 1764 & 1.2 & GTX 1060 \\
18 & 2019 & ICCV & STRN & $56.99 \%$ & 188 & 228 & 9262 & 70725 & 908 & 13.8 & GPU (?) \\
19 & 2018 & IEEEAccess & FPSN & $56.52 \%$ & 184 & 230 & 7682 & 71089 & 3005 & 10.1 & Titan X \\
20 & 2019 & arXiv & HISP_DAL & $56.08 \%$ & 174 & 239 & 7944 & 71012 & 3642 & 3.2 & GTX 1050 \\
21 & 2019 & IEEEAccess & OTCD [19] & $55.81 \%$ & 159 & 295 & 3715 & 78160 & 1228 & 5.5 & Titan X \\
22 & 2020 & arXiv & TrctrD17 & $55.56 \%$ & 171 & 271 & 5247 & 77615 & 715 & 4.9 & Titan X \\
24 & 2019 & ICCV & Tracktor++ [11] & $55.32 \%$ & 169 & 269 & 5375 & 77868 & 789 & 1.5 & Titan X \\
\hline
\end{tabular}
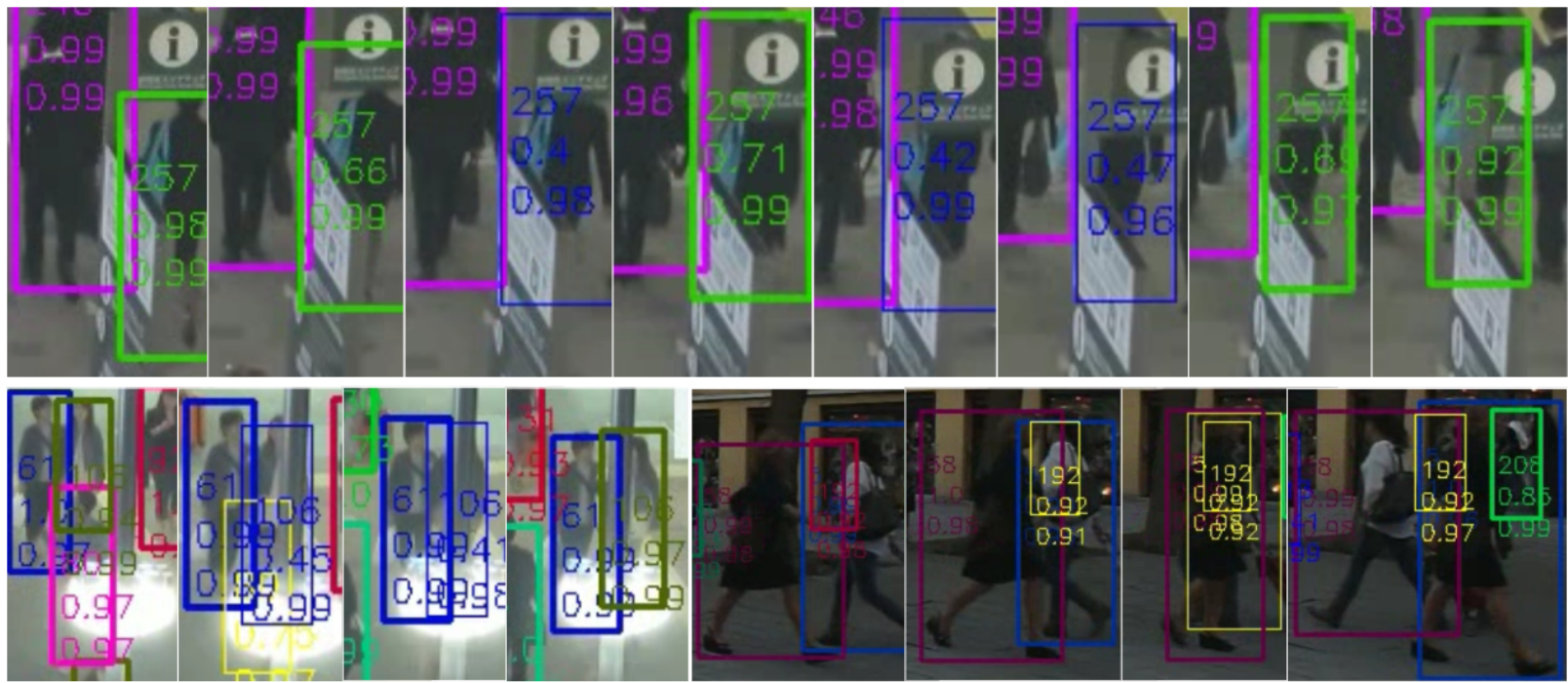

Fig. 3. Qualitative examples of our framework. Thick colored: identified tracklets associated with a detection. Thin blue: non-associated tracks being kept active because of their confidence score. Thin yellow: inactive tracks. In each box, the first number is the target identity, the second is the detection confidence score and the third is the tracklet reidentification score with the associated detection or predicted position. The first row and left half of the second one illustrate cases where keeping a confident track active pays off under scene occlusions. The right half of second row shows a type of identity switch our framework still struggles with. For better viewing, a video is provided as supplementary material.

knowledge. However, because this strategy filters out more tracklets, it still suffers from high false negatives.

$(A+D+E)$ On the other hand, keeping confident tracklets active even when no detection was assigned, and without any reidentification insight, tends to inevitably increase false positives - symmetrically decrease false negatives, and be less efficient on identity switches than with $(\mathrm{A}+\mathrm{E})$ configuration.

$(\mathbf{A}+\mathbf{B}+\mathbf{D}+\mathbf{E})$ As highlighted in table II, using both reidentification and keep active in a complementary fashion allows to both lower false negatives and identity switches while maintaining false positives relatively low. In the end, when $(\mathrm{A}+\mathrm{D}+\mathrm{E})$ impacts MOTA negatively and $(\mathrm{A}+\mathrm{B}+\mathrm{E})$ shows a small gain of $0.41 \%$, combining them leads to a $0.78 \%$ gain.

3) Benefiting from raw detector inputs: $(\mathrm{A}+\mathrm{B}+\mathrm{C}+\mathrm{D}+\mathrm{E})$ Adding detector confidence scores into the pipeline leads to a global gain of $0.96 \%$ on the MOTA, and $0.18 \%$ relatively to the previous configuration $(\mathrm{A}+\mathrm{B}+\mathrm{D}+\mathrm{E})$. In table II, we observe a rebalance on each type of error but more specifically on the false positive part, confirming the fact that tracker and detector are complementary mechanisms and can both benefit from each other.

Not illustrated in this paper, we also led experiments applying a threshold on upcoming detection scores to only consider trusted ones, it decreases the MOTA and confirms our first 
belief of tracker-detector complementarity.

4) Substantial gains: The gains presented in this ablation study are substantial regarding the challenge: comparing our method relatively to the other approaches in the literature (table III), we note that improving our method by almost +1.0 in MOTA led to a 6 place jump forward in the overall ranking.

Figure 3 shows qualitative examples of our framework The first row as well as the first half of second row show cases benefiting from our keep active strategy described in section III-C3. Looking at tracklets 257 and 106, we observe a balance between detector and tracker, where detection confidence scores start to drop under occlusions with objects in the scene but where tracks are still being kept active thanks to a reidentification score (hence a confidence score) remaining high. The tracker fulfils its catch-up role when the detector fails.

However, when total occlusions occur between targets (purple and blue targets on the right half of second row), the system deactivates the occluded track and struggles associating it correctly when it appears again. As illustrated in the second thumbnail, this is mainly because before deactivating the background track, a large portion of the targets gets involved in the two appearance feature vectors, leading to miss-reidentification.

\section{MOT17 evaluations}

In table III we compare our framework to the literature on the MOT17 test set with SDP detections. For fair comparison (especially on the execution time), we only show the CNNbased approaches, whether they use deep networks for position prediction or for reidentification. In any case, they generally perform better than other methods, with only one or two exceptions.

A few approaches integrating SOT for position prediction are designed concurrently with our work [9], [23], but we still present an original all-in-one architecture which obviously outperforms recent state-of-the-art MOTA performance on the benchmark [11], [17]. Beside tracking performance, we put forward a $\times 2$ to $\times 17$ gain in speed w.r.t. the the MOTA topranked CNN-based approaches, putting our method on the path towards realtime constraints for embedded applications.

\section{CONCLUSION AND FUTURE WORKS}

In this work, we put forward an original architecture integrating a jointly trained all-in-one single light-weight siamese $\mathrm{CNN}$ for both tracklet position prediction and reidentification in a multi-object tracking context. We present a novel training data generation and tracklet management strategy for this purpose and show state-of-the-art results on the well-known MOT17 benchmark, in terms of both tracking results and speed.

Due to the success of recent single object tracking work using intercorrelation inside their network [5], [24], [25], we aim at improving our architecture with such techniques but also find an in-between strategy within the appearance feature management strategy to deal with identity switches induced by full target-to-target occlusions.

\section{REFERENCES}

[1] A. Milan et al., "MOT16: A benchmark for multi-object tracking," 2016. [Online]. Available: http://arxiv.org/abs/1603.00831 1, 2, 6

[2] Y. Taigman et al., "DeepFace: Closing the gap to human-level performance in face verification," IEEE Conference on Computer Vision and Pattern Recognition (CVPR), 2014. 1

[3] F. Schroff et al., "FaceNet: A unified embedding for face recognition and clustering," IEEE Conference on Computer Vision and Pattern Recognition (CVPR), 2015. 1

[4] D. Held et al., "Learning to track at $100 \mathrm{fps}$ with deep regression networks," in European Conference on Computer Vision (ECCV), 2016. $1,3,6$

[5] B. Li et al., "High Performance Visual Tracking with Siamese Region Proposal Network," IEEE Conference on Computer Vision and Pattern Recognition (CVPR), 2018. 1, 8

[6] Y. Xu et al., "SiamFC++: Towards Robust and Accurate Visual Tracking with Target Estimation Guidelines," AAAI Conference on Artificial Intelligence, 2020.

[7] M. Kristan et al., "A novel performance evaluation methodology for single-target trackers," IEEE Trans. Pattern Anal. Mach. Intell., 2016.

[8] P. Chu and H. Ling, "Famnet: Joint learning of feature, affinity and multi-dimensional assignment for online multiple object tracking," in IEEE International Conference on Computer Vision (ICCV), 2019. 1, 2,7

[9] W. Feng et al., "Multi-Object Tracking with Multiple Cues and Switcher-Aware Classification," 2019. [Online]. Available: http://arxiv. org/abs/1901.06129 1, 2, 3, 4, 7, 8

[10] M. Tan and Q. V. Le, "Efficientnet: Rethinking model scaling for convolutional neural networks," in International Conference on Machine Learning (ICML), 2019. 1

[11] P. Bergmann et al., "Tracking without bells and whistles," in 2019 IEEE International Conference on Computer Vision (ICCV), Seoul, Korea, Oct. 2019. 1, 2, 3, 4, 5, 7, 8

[12] L. Chen et al., "Real-Time Multiple People Tracking with Deeply Learned Candidate Selection and Person Re-Identification," IEEE International Conference on Multimedia and Expo (ICME), 2018. 1, 2, 3 , 7

[13] A. Bewley et al., "Simple online and realtime tracking," in IEEE International Conference on Image Processing (ICIP), 2016. 2

[14] R. Sanchez-Matilla et al., "Online multi-target tracking with strong and weak detections," in European Conference on Computer Vision Workshop (ECCVW, 2016. 2

[15] N. Wojke et al., "Simple online and realtime tracking with a deep association metric," in IEEE International Conference on Image Processing (ICIP), 2017. 2, 3, 4, 5

[16] "Learning discriminative appearance models for online multi-object tracking with appearance discriminability measures," 2020. 2

[17] J. Zhu et al., "Online multi-object tracking with dual matching attention networks," in European Conference on Computer Vision (ECCV, 2018. 2,8

[18] S. Karthik et al., "Simple unsupervised multi-object tracking," 2020. [Online]. Available: http://arxiv.org/abs/2006.02609 2

[19] Q. Liu et al., "Real-time online multi-object tracking in compressed domain," IEEE Access, 2020. 3, 7

[20] J. Munkres, "Algorithms for the assignment and transportation problems," Journal of the Society for Industrial and Applied Mathematics, vol. 5, no. 1, 1957. 3

[21] F. Hutter et al., "Sequential model-based optimization for general algorithm configuration," in International Conference on Learning and Intelligent Optimization (LION), 2011. 6

[22] K. Bernardin and R. Stiefelhagen, "Evaluating multiple object tracking performance: The clear mot metrics," EURASIP Journal on Image and Video Processing, vol. 2008, 01 2008. 6

[23] Q. Chi et al., "Dasot: A unified framework integrating data association and single object tracking for online multi-object tracking," $A A A I$ Conference on Artificial Intelligence (AAAI), 2020. 7, 8

[24] Z. Zhu et al., "Distractor-aware siamese networks for visual object tracking," in European Conference on Computer Vision (ECCV), September 2018. 8

[25] B. Li et al., "Siamrpn++: Evolution of siamese visual tracking with very deep networks," in IEEE Conference on Computer Vision and Pattern Recognition (CVPR), 2019. 8 